Aim To assess whether ADHD symptoms and relational problems are associated with changes in entorhinal cortical thickness in very low birth weight (VLBW) adolescents.

Design/methods Fifty VLBW (birth weight $\leq 1500 \mathrm{~g}$ ) and 57 term control adolescents were assessed at 14-15 years of age with: ADHD Rating Scale IV (mother report), Strengths and Difficulties Questionnaire (SDQ mother report), Child Behaviour Check List (CBCL mother report) and Autism Spectrum Screening Questionnaire (ASSQ). Entorhinal cortical thickness (mm) was obtained using an automated MRI segmentation technique (Freesurfer). Associations were analysed by linear regression, adjusted for age, gender and socioeconomic status, and corrected for multiple comparisons (Benjamini-Hochberg procedure).

Results VLBW adolescents had higher ADHD and ASSQ scores than controls. On MRI, they had thinner entorhinal cortex compared to controls. Thinner entorhinal cortex was associated with higher ASSQ scores (Left: $\mathrm{B}=-0.946(-1.517$ to -0.374$), \mathrm{p}=$ 0.002 ; Right: $\mathrm{B}=-0.759(-1.308$ to -0.210$), \mathrm{p}=0.008)$, and higher SDQ Peer Problems Scale scores (Right: $\mathrm{B}=-0.254$ (0.459 to -0.050$), \mathrm{p}=0.016)$.

Conclusion Relational problems were associated with entorhinal cortical thinning in adolescents born preterm with VLBW, while associations were not found for ADHD symptoms. The different association in the two symptom groups with the entorhinal cortex might help to identify deviant neural structures and their relation to specific mental disorders.

\section{PO-0005 CEREBELLUM, THALAMUS AND CEREBRAL CORTEX IN VLBW ADOLESCENTS' MENTAL HEALTH}

${ }^{1} \mathrm{~V}$ Lozano-Botellero, ${ }^{1} \mathrm{MS}$ Indredavik, If Skranes, ${ }^{2} \mathrm{~S}$ Lydersen, ${ }^{2} \mathrm{AM}$ Brubakk, ${ }^{2} \mathrm{M}$ Martinussen. 'Laboratory Medicine Children's and Women's Health, Norwegian University of Science and Technology, Trondheim, Norway; ${ }^{2}$ Regional Centre for Child and Youth Mental Health and Child Welfare, Norwegian University of Science and Technology, Trondheim, Norway

\subsection{6/archdischild-2014-307384.686}

Background Children born preterm present a higher risk of psychiatric disorders during adolescence.

Aim To assess whether psychiatric symptoms are associated with changes in cortical thickness and volumes of thalamus and cerebellum in very low birth weight (VLBW) adolescents.

Design/methods Fifty VLBW (birth weight $\leq 1500 \mathrm{~g}$ ) and 57 term control adolescents were assessed at 14-15 years of age with: Schedule for Affective Disorders and Schizophrenia for School-Age Children, Strengths and Difficulties Questionnaire (SDQ Mother Report), Autism Spectrum Screening Questionnaire (ASSQ), and Children's Global Assessment Scale (CGAS). Cortical thickness $(\mathrm{mm})$ and volumes of thalamus and cerebellum (ml) were obtained using an automated MRI segmentation technique (Freesurfer). Associations were analysed by linear and ordinal logistic regression, adjusted for age, gender and total intracranial volume, and corrected for multiple comparisons (Benjamini-Hochberg).

Results VLBW adolescents had more psychiatric symptoms and diagnoses than controls. On MRI, they had several areas with thinner cortex, including the entorhinal cortex, and areas of thicker cortex, including the insula, compared to controls. Higher SDQ Emotional symptoms scores were associated with thicker insular cortex (Left: $\mathrm{B}=0.418$ (0.192 to 0.644), $\mathrm{p}=$ 0.001 ; Right: $\mathrm{B}=0.243$ (0.061 to 0.426$), \mathrm{p}=0.010)$. Smaller cerebellar WM volumes were associated with higher SDQ Hyperactivity scores (Left: $\mathrm{B}=-0.638$ (-1.101 to -0.176$), \mathrm{p}=$
0.008; Right: $\mathrm{B}=-0.551$ (-0.966 to -0.137$), \mathrm{p}=0.010)$ and lower CGAS scores (Left: $\mathrm{B}=4.653$ (2.182 to 7.123), $\mathrm{p}<0.001$; Right: $\mathrm{B}=4.255$ (2.073 to 6.437), $\mathrm{p}<0.001$ ).

Conclusion Our results indicate that psychiatric symptoms in VLBW adolescents may be related to structural brain anomalies in cerebellar white matter and insular cortex.

\section{PO-0006 WITHDRAWN}

\section{PO-0007 KNOWLEDGE AND ATTITUDE OF ADOLESCENTS TOWARDS HIVIAIDS-A CROSS-SECTIONAL STUDY}

P Dutta Kukreja. PICU, Great Ormond Street Hospital, London, UK

\subsection{6/archdischild-2014-307384.687}

Introduction Adolescents form a sizeable portion of the Indian population. In India, AIDS prevention and control efforts remained largely concentrated on groups already practising highrisk behaviour (like CSWs, iv drug abusers, etc.). Thus other potential groups like older school children, adolescents and younger adults, who because of their vulnerability deserve simultaneous attention, continue to remain a low priority.

Methods The present study was carried out to assess the level of awareness among school and college going students between the ages of 14-19 years about HIV/AIDS in Assam, India. 500 adolescents were given a pretested questionnaire and requested to fill it up and return within half an hour. The data was analysed manually using tally mark method and also subjected to Chi Square test of independent analysis and proportion test wherever needed.

Results The main source of HIV/AIDS awareness was media with very little information obtained from parents and teachers, representing that matters pertaining to sex, sexuality and reproduction still continue to be a taboo in our society. Misbeliefs on the modes of transmission of the disease like through handshake, kissing, use of fomites was close to $30 \%$.

There is also a minor discrepancy between knowledge and attitude. Though majority of the study population had a good knowledge of HIV/AIDS, a substantial portion (20-30\%) demonstrated negative attitude towards HIV/AIDS victim.

Conclusion A more appropriate programme based on behavioural science is desirable to lessen discrepancies between knowledge and desirable attitude along with Implementation of HIV/ AIDS awareness programme in schools, colleges and community.

\section{PO-0008 PULMONARY TUBERCULOSIS IN A 4 MONTH OLD WITH NO RESPIRATORY SYMPTOMS}

S Osman, A Hamad, S Khan, M Henduas, S Elhassan. General Pediatrics, Hamad Medical Corporation, Doha, Qatar

\subsection{6/archdischild-2014-307384.688}

Background Fever of unknown (FUO) origin often presents a unique diagnostic challenge to the paediatrician. We present a unique case of a 4 month old boy with fever for 1 month, who after extensive work up was diagnosed with pulmonary tuberculosis and Severe Combined Immunodeficiency (SCID).

Case Report Our case is a 4 month old, Sudanese boy, residing in Qatar, who presented to our hospital with fever for two weeks. He remained febrile in the hospital for two weeks, 